TU1D-5

\title{
Nonlinear Transmission Lines in Left-Handed Media
}

\author{
Alexander B. Kozyrev and Daniel W. van der Weide \\ Department of Electrical and Computer Engineering, The University of Wisconsin-Madison, \\ Madison, Wisconsin 53706, USA
}

\begin{abstract}
We introduce nonlinear transmission lines (NLTL) based on left-handed (LH) media and simulate thirdharmonic generation in a material that in two dimensions could also focus microwaves. We discuss physical phenomena that lead to and affect harmonic generation in LH NLTL and we outline advantages of LH NLTL media for developing a new type of frequency multiplier.
\end{abstract}

Index Terms - frequency conversion, high-pass filter, left-handed media, metamaterials, negative refractive index, nonlinear transmission line, periodic structure

\section{INTRODUCTION}

Artificial materials (metamaterials) with simultaneously negative permeability and permittivity are sometimes called left-handed materials (LHM). LHMs use arrays of metallic wires and arrays of split-ring resonators [1] or planar transmission lines periodically loaded with series capacitors and shunt connected inductors [2], [3]. The unique electrodynamic properties of these materials, first predicted by Veselago in 1968 [4], include the reversal of Snell's law, Doppler effect, Cherenkov radiation and negative refractive index, making them attractive for fabrication of new types of radio frequency ( $\mathrm{f}$ ) and microwave components. The most tantalizing is the possibility to use these materials to build perfect lenses because of their inherent negative refractive index [5].

Most studies of LHM have been performed in linear regime of wave propagation. However, combination of nonlinearity and anomalous dispersion of LHM may give rise to many new and interesting phenomena and applications. Some nonlinear wave phenomena that occur during propagation of the wave along the boundary between right-hand medium (RHM) and LHM, when one or both of them are nonlinear, have been considered in [6]-[9]. Here we consider for the first time nonlinear wave phenomena in LHM alone. We present simulations of higher harmonic generation in LHM based on the dual of the conventional nonlinear transmission line (NLTL) or left-handed (LH) NLTL with anomalous dispersion. Such structures can be realized with varactor diodes instead of capacitors in the transmission line negative refractive index media proposed by Eleftheriades et al. [2]. The mechanism of higher harmonic generation is nonlinear resonance in the LH NLTL, which results in self-induced periodicity leading to self-induced phase-matching of the fundamental wave with one of its higher harmonics.

\section{MODEL DESCRIPTION}

The equivalent circuit of the LH NLTL used in our simulations is shown in Fig. 1. Nonlinear wave processes in this equivalent circuit are governed by the following system of equations:

$$
\begin{gathered}
C_{n} \frac{d}{d t}\left(V_{n}-V_{n-1}-R_{d} i_{n}^{C}\right)=i_{n}^{C} \\
L_{0} \frac{d}{d t}\left(i_{n+1}^{C}-i_{n}^{C}\right)=V_{n},
\end{gathered}
$$

where $L_{0}$ is the inductance, $R_{d}$ is the diode series resistance, $V_{n}$ and $i_{n}^{C}$ are respectively the voltage at the $n$-th node and current through $n$-th nonlinear capacitor $C_{n}=C\left(V_{n}-V_{n-1}\right)$ formed by two back-to-back varactor diodes to provide symmetric capacitance-voltage characteristics expressed as:

$$
C(V)=\frac{C_{j 0}}{\left(1-\left|V / V_{j 0}\right|\right)^{M}}
$$

In our simulations the values for prototype Agilent TC803 hyperabrupt varactor diodes are used: $C_{j 0}=1 \mathrm{pF}$, $M=1.039, V_{j 0}=0.7 \mathrm{~V}, R_{d}=6 \Omega$. These diodes were chosen because of their high capacitance ratio: $C(0 \mathrm{~V}) / C(10 \mathrm{~V})=17$. The input port of LH NLTL is fed by sinewave signal $V_{i n p}=V_{0} \sin (\omega t)$. The amplitude of the input signal is chosen so that the voltage drop across the diodes does not exceed the breakdown voltage $V_{b r}=10 \mathrm{~V}$.

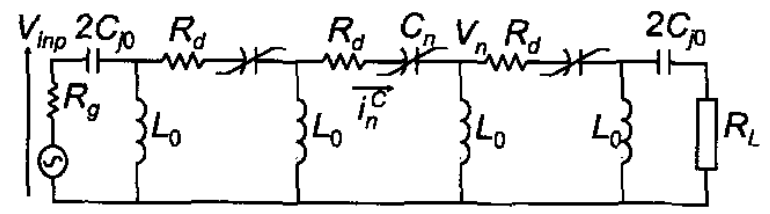

Fig. 1. Equivalent circuit of LH NLTL. 
The circuit is loaded with resistance $R_{L}=0.8 Z_{0}$ $\left(Z_{0}=\left(L_{0} / C_{j 0}\right)^{1 / 2}\right)$ which is equal to generator resistance $R_{\mathrm{g}}=R_{L}$. A simple 5-diode LH NLTL (only 3 diodes are shown in Fig. 1 for simplicity) has been simulated with the commercial microwave circuit simulator Agilent ADS.

In the linear case $\left(C_{n}=C_{j 0}\right)$ without losses $\left(R_{d}=0\right)$, the dispersion relation is:

$$
\omega^{2}=\frac{1}{4 L_{0} C_{j 0} \sin ^{2} \varphi / 2},
$$

where $\varphi$ is the phase shift per section. The value $\varphi=\pi$ corresponds to the boundary of the transparency band (the minimal frequency of the propagating wave or the Bragg cutoff frequency):

$$
\omega_{B}=\frac{1}{2 \sqrt{L_{0} C_{j 0}}}
$$

In the linear limit, the circuit under consideration is a high pass filter. When $\omega$ is increased, $\varphi$ decreases monotonically (anomalous dispersion). The fundamental wave $\left(-\pi \leq \varphi_{0} \leq \pi\right)$ propagating in this structure is backward (phase and group velocities are directed in opposite directions). This property permits us to refer this structure as left-handed (in Veselago's terminology [4]).

\section{RESULTS OF SIMULATIONS}

Fig. 2 plots conversion efficiency for $3^{\text {rd }}$ and $5^{\text {th }}$ harmonics (the ratio of the harmonic power delivered to the load to the input power applied to LH NLTL) together with the ratio of the power of the fundamental wave (input wave) transmitted to the load versus normalized frequency. Generation of higher harmonics is possible in a limited frequency range. From the lower side, the $3^{\text {rd }}$ harmonic generation region is limited by the transmission line Bragg cutoff frequency (5). At the same time due to the nonlinearity of the system, any voltage drop across the diodes will shift the cutoff frequency up. Strictly speaking, the wave can be considered as propagating only if its frequency is

$$
\omega>\omega_{*}=\frac{1}{2 \sqrt{L_{0} C\left(V_{\max }\right)}}
$$

where $V_{\max }=V_{b r}$ is the maximum voltage across the varactor. Results of the simulations showed that the upper boundary of the $3^{\text {rd }}$ harmonic generation region roughly corresponds to this frequency. In our case $\omega_{*} / \omega_{B}=4.1$.
In the gap between the cutoff frequency and $\omega_{*}$ we should expect a monotonic growth of the power of the fundamental (input) wave transmitted to the load. We can see that growth in Fig. 2 together with the increase of the third-harmonic efficiency.

The maximum of $3^{\text {nd }}$ harmonic generation efficiency corresponds to the total phase shift across the LH NLTL of $2 \pi$ which we call a nonlinear resonance. Detailed analysis has demonstrated that an intense fundamental wave induces a periodic variation of the capacitance along the LH NLTL. The period of this variation is equivalent to two stages of LH NLTL. This self-induced periodicity results in a dramatic change of the dispersion characteristics, enabling phase-matching of the fundamental wave and its $3^{\text {rd }}$ harmonic, which would otherwise be impossible in linear limit. Harmonic generation in LHM is qualitatively different from harmonic generation in RHM. For example, in the conventional low-dispersive NLTL, the fundamental wave stays phase-matched with its $3^{\text {rd }}$ harmonic in the linear limit.

Furthermore, the periodicity (discreteness) of the LH NLTL is essential for harmonic generation. The periodicity is inherent to realizable LH NLTLs, but to get harmonic generation in quasihomogeneous, LHMs like those using arrays of metallic wires and split-ring resonators, one should load them periodically with nonlinear components.

The drop in power transmitted to the load when we move out of resonance is related to the instability known as parametric generation of traveling waves [10]. The high-frequency fundamental (pump) wave with

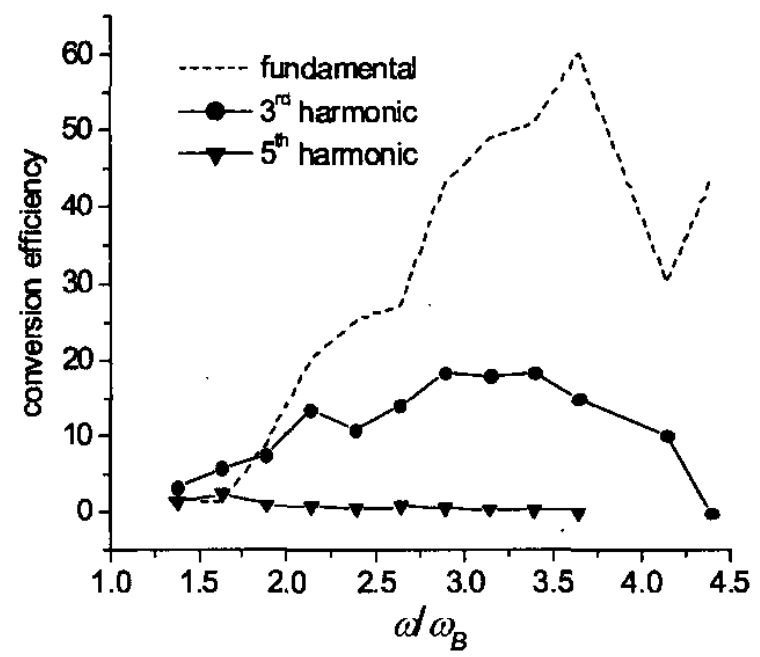

Fig. 2. Conversion efficiency (\%) vs. relative frequency. 
frequency $\omega_{0}$ generates three other waves with frequencies $\omega_{1}<\omega_{0}, \omega_{2}=2 \omega_{0}-\omega_{1}$ and $\omega_{3}=\omega_{0}+2 \omega_{1}$ so that the phase-matching condition for four interacting waves is satisfied. The wave at $\omega_{2}$ propagates in the opposite direction relative to the fundamental (pump) wave and the two other parametrically generated waves. We thus have a similar situation to backward wave parametric generation [10], [11]. Waveform evolution in the LH NLTL has many similarities with the competition between resonant excitations and nonlinear parametric interactions recently discussed in nonlinear optics (see [12] and references wherein).

Fig. 3 compares voltage waveforms at the input and at the load corresponding to the maximum $3^{\text {rd }}$ harmonic conversion efficiency and Fig. 4 is the spectrum of the latter waveform. The $5^{\text {th }}$ harmonic conversion efficiency is $\leq 1 \%$ so power conversion into the $3^{\text {rd }}$ harmonic is very efficient (about $20 \%$ ) and generation of higher harmonics is suppressed. Assurning $R_{L}$ to be $50 \Omega$, one can solve for circuit parameters specified in Section II, arriving at the value for the fundamental (input) frequency corresponding to the maximum conversion efficiency to be $3.7 \mathrm{GHz}$ and for the value of power of $3^{\text {rd }}$ harmonic delivered to the load to be $14 \mathrm{~mW}$.

The $3^{\text {rd }}$ harmonic conversion efficiency in LH NLTL is of the same order as that which can be obtained in a conventional periodically loaded NLTL with normal dispersion (of the low-pass filter type) [13], [14] but the

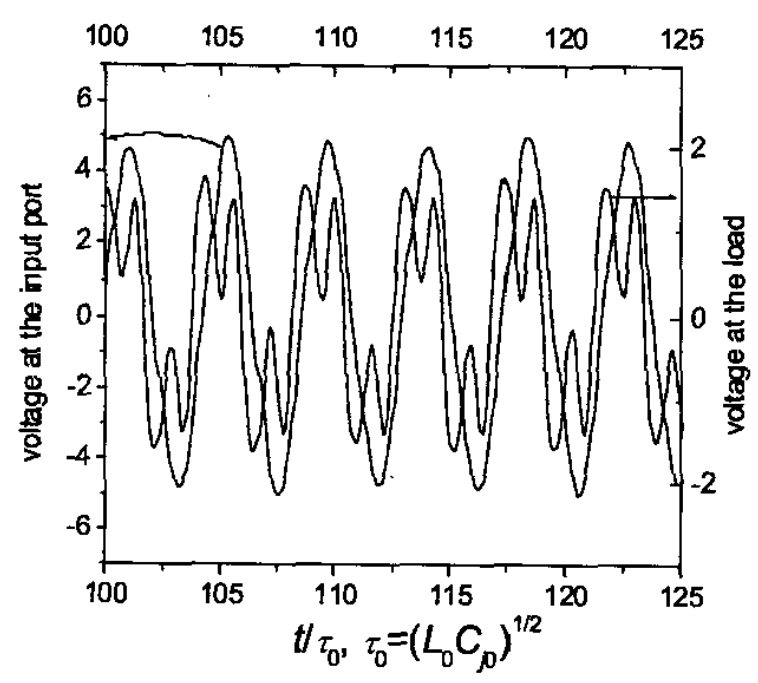

Fig. 3. Comparison of input and output waveforms corresponding to maximum of conversion efficiency $\left(\omega_{\text {inp }} / \omega_{B}=2.89\right)$. required transmission line length is shorter for the $\mathrm{LH}$ NLTL, so the per-section efficiency is higher. Also the 3$\mathrm{dB}$ operating bandwidth is two times wider ( $60 \%$ vs. $30 \%$ [13]). The efficiency is limited by the occurrence of parametric instability at high frequencies (which is determined by nonlinearity, namely, capacitance ratio and breakdown voltage) rather than the Bragg cutoff frequency. For the same reason, the frequency of the fundamental (input) wave can be higher for a given $C_{j 0}$ and $L_{0}$ in the LH NLTL.

Finally, we note that all the qualitative considerations presented here are valid for second harmonic generation in LH NLTL periodically loaded with biased diodes with asymmetric capacitance-voltage characteristics.

\section{CONCLUSIONS}

Our simulations demonstrate efficient harmonic generation along LH NLTLs. Harmonic generation is possible over a significantly wider operating frequency range and at relatively higher frequencies in comparison with dual conventional low-pass filter type NLTL. Extending these results for one-dimensional LH NLTL to two dimensions would enable combined harmonic generation in LH NLTL media with focusing, due to the negative refractive index of 2-D LH transmission line media, leading to the development of highly efficient powerful frequency multipliers.

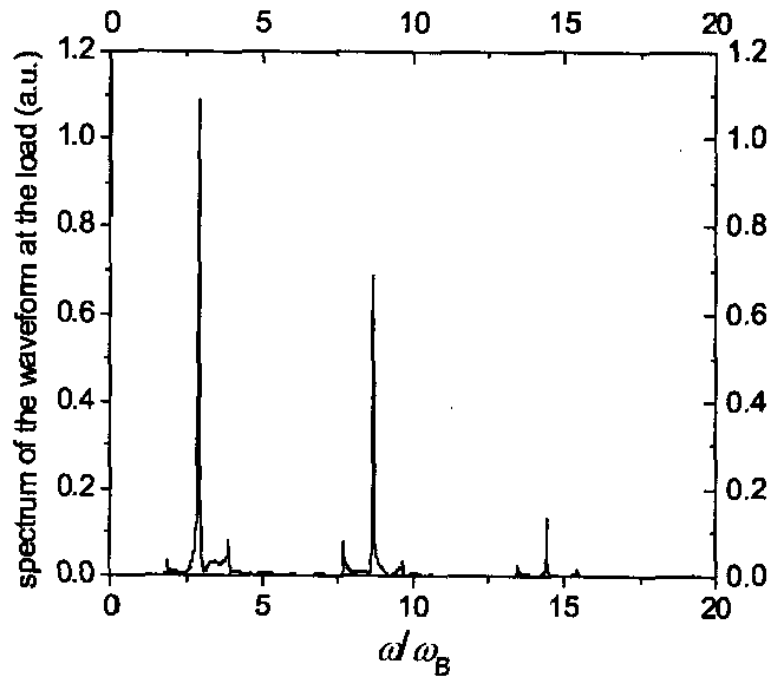

Fig.4. Spectrum of the voltage waveform at the load shown in Fig. 3. 


\section{ACKNOWLEDGEMENT}

This work is supported by the AFOSR through the MURI program.

\section{REFERENCES}

[1] D. R. Smith, W. J. Padilla, D. C. Vier, S. C. Nemat-Nasser, and S. Schultz, "Composite medium with simultaneously negative permeability and permittivity", Phys. Rev. Lett., vol. 84 , no. 18 , pp. 4184-4187, May 2000.

[2] G. V. Eleftheriades, A. K. Iyer, and P. C. Kremer, "Planar negative refractive index media using periodically L-C loaded transmission lines", IEEE Trans. Microwave Theory \& Tech., vol. 50, no. 12, pp. 2397-2402, December 2002.

[3] A. Grbic and G. V. Eleftheriades, "Experimental verification of backward-wave radiation from a negative refractive index metamaterial", J. Appl. Phys., vol. 92, no 10 , pp. 5930-5935, 15 November 2002.

[4] V. G. Veselago, "The electrodynamics of substances with simultaneously negative values of $\mathrm{e}$ and $\mathrm{m}, "$ Sov. Phys.Usp., vol. 10, no. 4, pp. 509-514, Jan.-Feb. 1968.

[5] R. A. Shelby, D. R. Smith, and S. Schultz, "Experimental verification of a negative index of refraction," Science, vol. 292, pp. 77-79, Apr. 2001.

[6] A. M. Belyantsev, A. B. Kozyrev, "RF oscillation generation in coupled transmission lines with anomalous and normal dispersion", Technical Physics, vol. 46, no. 7, pp. 864-867, 2001.
[7] A. B. Kozyrev, "The structure of a shock electromagnetic wave synchronous with several waves propagating in coupled transmission lines with different types of dispersion", Technical Physics, vol. 47, no. 2, pp. 272-274, 2002.

[8] A. M. Belyantsev, A. B. Kozyrev, "Reversed Doppler effect under reflection from a shock electromagnetic wave", Technical Physics, vol. 47, no. 11, pp. 1477-1480, 2002.

[9] I. V. Shadrivov, A. A. Sukhorukov, Y. S. Kivshar, "Nonlinear surface waves in left-handed materials", Phys. Rev. $E$, vol. 69 , no. 1, p. 016617 , January 2004.

[10] A. S. Gorshkov, G. A. Lyakhov, K. I. Voliak, L. A Yarovoi, "Parametric generation in anomalously dispersive media", Physica D, vol. 122, pp. 161-177, 1998.

[11] S. E. Harris, "Proposed backward wave oscillation in the infrared", Appl. Phys. Lett., vol. 9, no. 3, pp. 114-116, 1 August 1966.

[12] R. Frey, "Suppression of the medium excitation in resonant nonlinear optics", Opt. Comm., vol. 89, no. 5-6, pp. 441446, 15 May 1992.

[13] J. Duchamp, P. Ferrari, M. Femandez, A. Jrad, X. Melique, J. Tao, S. Arscott, D. Lippens, and R. G. Harrison, "Comparisom of fully distributed and periodically loaded nonlinear transmission lines", IEEE Trans. Microwave Theory \& Tech., vol. 51, no. 4, pp. 1105-1116, April 2003.

[14] M. J. Rodwell, S. T. Allen, R. Y. Yu, M. G. Case, U. Bhattacharya, M. Reddy, E. Carman, M. Kamegawa, Y. Konishi, J. Pusl, and R. Pullela, "Active and nonlinear wave propagation in ultrafast electronics and optoelectronics," Proc. IEEE, vol. 82, pp. 1037-1059, July 1994. 